\title{
ESTADO EMOCIONAL DE NIÑOS Y ADOLESCENTES AFECTADOS POR EL INVIERNO
}

\section{Child and adolescent emotional state affected by winter}

Leslie Yuliet Bravo García*, Janelly Andrea Bustamante Benítez**, Mónica Isabel Herazo Chamorro***, Rubiela Godin Díaz****, Yira Meléndez Monroy*****

\section{Resumen}

Colombia padeció una fuerte temporada invernal durante el año 2010 y parte del 2011, producto del Fenómeno de la Niña. Por tanto se pretendía describir el estado emocional de los niños, niñas y adolescentes (NNA) afectados por la ola invernal de 2011 en las subregiones Mojana y San Jorge del departamento de Sucre, hallándose estos entre los 10 y los 14 años y utilizando una muestra de 406 NNA para el estudio. La metodología fue de paradigma positivista, de tipo descriptivo, encontrando que los NNA no poseen defensas suficientes para adaptarse al ambiente, en un $88 \%$ en Mojana y 95\% en San Jorge, lo cual sugiere una pronta intervención a la infancia y adolescencia afectada por cambios climáticos.

Palabras clave: Estado emocional, ola invernal, emociones, infantes, adolescentes.

\begin{abstract}
Columbia suffered a strong winter season during 2010 and part of 2011, as a result of the "La Niña" Phenomenon. Thus, in attempt to describe the emotional state of children and adolescents affected by the harsh winter of 2011 in the Sub Regions Mojana and San Jorge from the department of Sucre, a sample of 406 children and adolescents, aged between 10 and 14 years, were studied. The methodology used was descriptive and utilized a positivist paradigm, finding that the children and adolescents did not possess sufficient defenses in order to adapt to the environment, 88\% in Mojana and 95\% in San Jorge, which suggests a prompt intervention in infancy and adolescence affected by climate change.
\end{abstract}

Keywords: Emotional state, harsh winter, emotions, childhood, adolescents

\footnotetext{
* Psicóloga. Docente. Corporación Universitaria del Caribe. CECAR. leslie.bravo@cecar.edu.co

** Psicóloga. Corporación Universitaria del Caribe. CECAR. Janelly.bustamante@ cecar.edu.co

*** Psicóloga. Corporación Universitaria del Caribe. CECAR. Colombia. Monica.herazo@cecar.edu.co.

**** Trabajadora Social. Corporación Universitaria del Caribe. CERCAR. Colombia.

****** Psicóloga. Docente Corporación Universitaria del Caribe. CERCAR. Colombia.
} 


\section{INTRODUCCIÓN}

Colombia es el tercer país en el mundo que presenta más muertos por desastres o fenómenos naturales (Galindo, Pacheco y Russo, 2011) y entre los años 2010-2012 padeció una fuerte temporada invernal, la cual se denomina fenómeno de la niña y se caracteriza por una intensificación de la temporada de lluvia, sin embargo para este laxo de tiempo el fenómeno produjo mayores emergencias en relación a otros años, además de ser la catástrofe natural más devastadora en la historia más reciente de Colombia. El resultado de este fenómeno según las cifras que reporta el DANE (2011) fueron de 2.350.207 personas damnificadas, 869.032 personas afectadas, 647.017 hogares damnificados, 232.525 hogares impactados y 6.474 fallecidos, así mismo las emergencias presentadas fueron de 2.219, siendo 1.233 inundaciones, 778 deslizamientos, 174 vendavales, 24 avalanchas y los 10 eventos restantes se componen de tormentas eléctricas, granizadas y tornados (Comisión Económica para América Latina y el Caribe - CEPAL, 2012).

Las subregiones Mojana y San Jorge anualmente presentan inundaciones con duraciones promedio de 2 a 3 meses, pero para el 2010 las inundaciones se intensificaron y persistieron por más de 7 meses, con niveles de 2 y 3 metros nunca antes alcanzados, afectando 27.944 familias, 102.177 personas y 19.901 viviendas (Informe Ola invernal Sucre, 2011). En ese mismo informe se muestra que hubo daños en infraestructura, en cuanto a viviendas, escuelas, vías, y puentes; en la economía de ambas porque los cultivos de arroz se perdieron a causa de la inundación, reduciendo las actividades agropecuarias de las dos subregiones. Al igual las condiciones de bienestar para el 2010 fueron de $54.86 \%$ en NBI (necesidades básicas insatisfechas) y un $69.46 \%$ de población bajo la línea de pobreza (Plan de Desarrollo departamental de sucre, 2012 - 2015).

Considerando que la población de NNA por sus edades son más vulnerables a los cambios climáticos, su salud y desarrollo integral se ve afectado agudizando su problemática fundamentando, primero que no hay estadística de total de NNA afectados por el invierno, además esta población se halla en situación de "discapacidad, desplazamiento, víctimas del conflicto armado y de desastres naturales como las inundaciones", tercero la poca implementación del código de infancia adolescencia, cuarto en el indicador de atención a la infancia y adolescencia en el 2007 el plan de desarrollo departamental muestra que se hallaba en cero (Plan de desarrollo departamental de sucre 2008-2011) y al 2011 aún no se conocía avance luego de pasar la ola invernal y por último la ayuda psicológica prestada es precaria durante y después de la ola invernal lo cual genera mayores perturbaciones de tipo emocional en los NNA (Galindo et al. 2011).

Por tanto es necesario describir el estado emocional de estos NNA afectados por el invierno, porque las emociones regulan la forma en cómo los sujetos asimilan la realidad, adaptan al ambiente y como se relacionan con los demás, facilita la aparición de conductas apropiadas y orientan a esta para lograr los objetivos y metas que poseen (Choliz, 2005). Por ello si no se goza de un estado emocional totalmente saludable a causa de experiencias traumáticas, el individuo pierde los deseos de superación y presenta sentimientos de frustración y estancamiento (Choliz, 2005).

El fenómeno de la ola invernal o los desastres naturales crea incertidumbre, provoca inseguridad y da a lugar a diversas variantes traumáticas que es difícil de superar para el individuo, que si no se curan o superan, producen consecuencias negativas en la conducta, emociones, y psique (Galindo et al. 2011). Además desde una óptica de la salud mental, las emergencias y los desastres naturales implican una perturbación emocional que sobrepasa la capacidad de tratamiento y afrontamiento de la población afectada (Díaz, Castro y González, 2010; Leiva y Baher, 2012). En ese mismo sentido los NNA presentan mayores problemas de adaptación al ambiente que se encuentran inmersos, debido a que ocurre una fijación psicológica y la experiencia traumática se hace presente en el sujeto en su diario vivir, además experimentan estrés, ansiedad, angustia, terror, depresión, entre otras perturbaciones emocionales, que representan gran carga de estrés psicológico y pérdidas de todo tipo para el individuo (Díaz et al. 2010; Galindo et al. 2011). Al igual al estar los NNA en plena formación de su personalidad son más vulnerables a la situación que deben enfrentar en lo dicho por Bandura (2006), (citado por Guerra, Cumsille, y Martínez, 2014), la cual representa gran 
carga de estrés psicológico y pérdidas de todo tipo para el individuo (Díaz et al. 2010).

Entre los aspectos pertinentes de esta investigación se hallan primero que en el departamento de Sucre no hay investigaciones que hayan evaluado el estado emocional psicológico de los NNA afectados por el invierno y son escasas las que tratan es temática (García, 2011; Leiva y Baher, 2012), segundo las investigaciones que se han desarrollado en población infantil afectada por desastres naturales priorizan el aspecto psicopatológico (Cardozo, Martínez, y Colmenares, 2012), además estos estudios en su mayor parte se han hecho con población adulta (Guerra et al. 2014). Tercero se aporta conocimiento científico en tres aspectos importantes, el primero en la "representación psicológica mediante instrumentos proyectivos en la literatura de desastres naturales" (Díaz et al 2010), segundo a la psicología de las emergencias que ha sido poco abordada, y tercer a partir de lo anterior desarrollar intervenciones en el marco de la salud mental de esta población (Colmenares, 2011, citado por Cardozo et al, 2012).

Por ultimo esta investigación al hallarse adscrito a un proyecto institucional de la Corporación universitaria del Caribe - CECAR, es el aporte para la ruta de atención dentro del componente psicosocial del "protocolo de atención integral a la infancia y adolescencia afectada por eventos climáticos, en el departamento de sucre", así como la línea de base de lo que será el observatorio de infancia y adolescencia departamental.

\section{TEORÍAS PSICOANALÍTICAS DESARROLLO DE LAS EMOCIONES}

DEL

Con el psicoanálisis Freud introduce un agregado al concepto de emoción en el que se incluyen ideas fisiológicas y psicológicas de la modernidad. Así mismo, toma las premisas de Hume, que la emoción no es un evento mental o (first impression), sino una impresión secundaria o (reflective impression). Pero la diferencia radica en que esta impresión secundaria no deriva de ninguna impresión original (ni directamente, ni siquiera mediante la interposición de una idea) pues la causa de la emoción no tiene nada que ver ni con la conciencia ni con el cogito (Freud, 1915). La emoción es considerada entonces un fenómeno consciente, claro, pero que esconde una realidad engañosa en la que se oculta la causa real de la misma. En este sentido, Freud plantea que la fuente de la emoción, como también de los demás fenómenos de conciencia, es el Ello. El cual, posee un impulso especial, la líbido o pulsión instintiva más vaga y añeja. Es así, que se considera entonces a la emoción con dos elementos: el primero corresponde a las llamadas descargas físicas, y el segundo a ciertos sentimientos.

Teniendo en cuenta la descripción de Freud acerca deldesarrollodelas emociones seestablecela modalidad defensiva de los niños, niñas y adolescentes, y se toma entonces como referente a Anna Freud (1998), para la cual los mecanismos de defensa son una actividad del Yo que tiene como finalidad proteger a la persona de una excesiva intensidad pulsional y disminuir su tensión interna. Actúan inconscientemente, y son utilizados tanto por los sujetos normales como los neuróticos, pero en estos últimos con un carácter más patológico.

Anna Freud (1998) en su libro El Yo y los Mecanismos de defensa, se encarga de describir entre otras cosas, los recursos protectores más recurrentes que utiliza el niño, el adolescente y el adulto en su búsqueda de placer y evitación del miedo y la angustia, que en ocasiones conducen a la enfermedad. Dichos recursos protectores son la represión, aislamiento, anulación, introyección, vuelta contra sí mismo, transformación de lo contradictorio, sublimación, regresión, proyección, y la formación reactiva.

Todos estos mecanismos suelen soltar la tensión, incluso pueden algunos de ellos resolver más o menos aparentemente un problema o producir una satisfacción sustitutiva. También pueden ayudar a no desesperarse por las pocas cosas que pueden lograrse en proporción a los deseos y pueden hacer olvidar acontecimientos o hechos vividos que pueden considerarse dentro de lo absurdo.

\section{METODOLOGÍA}

El estudio adoptó una metodología de paradigma positivista de diseño cuantitativo de tipo descriptivo, donde la población fue de 19.383 Infantes y 
adolescentes afectados por la ola invernal en las subregiones Mojana y San Jorge del departamento de sucre que se hallaban entre los 10 -14 años de edad, donde la muestra a trabajar fueron 406 NNA que cumplía los criterios anteriores, dicha muestra fue el resultado de un muestreo estratificado en cada uno de los Municipios (Sucre - Sucre, Majagual, Guaranda, san Benito, Caimito y San Marcos) con un nivel de confianza del $95 \%$ y margen de error del $5 \%$, utilizando el programa Necquest.

La recolección de la información se hizo mediante la técnica del dibujo, utilizando el Test de Figura Humana y el Test Persona Bajo la Lluvia y sus análisis se hizo primero vaciando la información individual de cada uno de los evaluados por indicadores emocionales en unos cuadros de categoría, a los que se les asignó códigos a los niños evaluados para hacer el análisis de forma individual, después manualmente se sistematizó el número de indicadores emocionales de temores, conflictos, aspectos conductuales además de la modalidad defensiva y adaptativa en la que coincidieron los NNA.

La tabulación de la información se realizó empleando una hoja electrónica de Excel para la cuantificación de los resultados y el establecimiento de porcentajes en cada uno de los indicadores, los porcentajes se aproximaron a los enteros anteriores o siguientes según el caso.

\section{RESULTADOS}

A continuación se relacionan los resultados obtenidos de los indicadores más frecuentes en los NNA afectados, que permite hacer la descripción del estado emocional de ellos y comparar los resultados de las dos subregiones.
Tabla 1: Recursos expresivos del dibujo

\begin{tabular}{lrc}
\hline Indicador & Mojana \% N:192 & SanJorge \% N:214 \\
\hline Dimensión del dibujo & & \\
Normal & 48 & 10 \\
Pequeño & 50 & 73 \\
Grande & 1 & 16 \\
Emplazamiento & & \\
Margen superior & 4 & 14 \\
Margen izquierdo & 11 & 23 \\
Margen inferior & 1 & 14 \\
Centro de la hoja & 84 & \\
& & \\
Orientación de la persona & & 66 \\
Hacia la izquierda & 10 & 34 \\
Hacia el frente & 90 & \\
& & 34 \\
Sombreado & & 65 \\
Si & 9 & 17 \\
No & 91 & 83 \\
Borrados en el dibujo & & \\
Si & 3 & \\
No & 97 & \\
\hline
\end{tabular}

Nota: la elaboración de la tabla es propia. Adaptado de American Psychological Association. Manual de publicaciones de la American Psychological Association (6 ${ }^{\mathrm{a}}$ ed.). Mexico D.F.: Manual moderno

De acuerdo con lo tabla anterior se muestra que el porcentaje de dibujos medianos hechos por los NNA significa que estos se hallan ubicados bien en relación al espacio, y el porcentaje de dibujos pequeños indica que los NNA presentan temor de no reconocimiento, sensación de encierro o de incomodidad, inseguridad y retraimiento; conflictos porque la situación de presión ambiental genera en ellos sentimientos de inferioridad, inadecuada percepción de sí mismos, manifestándose conductualmente en estos NNA como tímidos, introvertidos, y dependientes. Reafirmando en su modalidad adaptativa los sentimientos de inadecuación. Los dibujos grandes muestran que el porcentaje de NNA que los realizaron tiene conflictos por falta de adaptación, y en su aspecto conductual se manifiesta como teatralidad, necesidad de mostrarse, de ser reconocido, de ser tenido en cuenta e índices de agresividad. 
Los dibujos emplazados en el centro de la hoja indican que el porcentaje de NNA que ejecutaron dibujos así, en su modalidad adaptativa muestran un criterio ajustado a la realidad, equilibrio entre tendencias de introversión y extroversión, objetividad, control de sí mismo, reflexión, y buen uso del espacio. Mientras que los NNA con dibujos emplazados en el margen superior, su aspecto conductual se caracteriza por rasgos de personalidad eufórica, alegre, noble, espiritual e idealista. Al igual los emplazados en el margen izquierdo indican que en estos NNA aparecen la debilidad y el desaliento ante el medio que le rodea, conflicto en cuanto a presentar depresión, y aspectos conductuales que les identifica como perezosos y presentar agotamiento en las actividades que realizan. Por su parte los dibujos emplazados en el inferior son NNA que se apegan a lo concreto, tienen tendencias instintivas muy marcadas, falta de imaginación que no deja que el individuo crezca espiritual y de forma psíquica.

En cuanto a la orientación en el dibujo los NNA que hicieron dibujos donde la persona se orienta hacia la izquierda tienen conflictos del pasado aún sin resolver y que aún le pesan, mostrándose conductualmente a través del bloqueo de la personalidad, de comportamiento y acciones que evidencian dependencia, e idealismo. Los dibujos con orientación hacia el frente muestra que los NNA en la modalidad adaptativa pese a las condiciones del ambiente se encuentran dispuestos a enfrentar el mundo, lo que permite inferir que estos se llegan a adaptar con mayor facilidad.

El sombreado en el dibujo indica en cuanto a conflictos que los NNA que lo hicieron sienten ansiedad por el cuerpo, necesidad de control sobre él mismo y en su modalidad de defensiva el mecanismo de defensa más utilizado es la anulación. Otro indicador como el de los borrados en el dibujo no se presentaron mucho, escaso fueron los NNA que hicieron borraduras, lo cual significa que este porcentaje de NNA tienen incertidumbre, en conflictos ansiedad, agresividad, y en aspecto conductual indecisión, descontrol, conductas agresivas.
Tabla 2: Contenido del dibujo

\begin{tabular}{|c|c|c|}
\hline Indicador & $\% \mathrm{~N}: 192$ & San Jorge \% N:214 \\
\hline \multicolumn{3}{|l|}{ Nubes } \\
\hline $\mathrm{Si}$ & 75 & 53 \\
\hline No & 26 & 48 \\
\hline \multicolumn{3}{|l|}{ Lluvia } \\
\hline $\mathrm{Si}$ & 62 & 38 \\
\hline No & 98 & 2 \\
\hline \multicolumn{3}{|l|}{ Tipo de lluvia } \\
\hline Lluvia torrencial & 38 & 34 \\
\hline Lluvia escaza & 50 & 22 \\
\hline Lluvia normal & 38 & 44 \\
\hline \multicolumn{3}{|l|}{ Gotas como lagrimas } \\
\hline $\mathrm{Si}$ & 42 & 54 \\
\hline No & 13 & 87 \\
\hline \multicolumn{3}{|c|}{ Botones, animales, flores, bolsillos } \\
\hline $\mathrm{Si}$ & 14 & 42 \\
\hline No & 85 & 58 \\
\hline \multicolumn{3}{|l|}{ Ojos } \\
\hline Sin pupila & 28 & 24 \\
\hline Como puntos & 10 & 6 \\
\hline Normal & 61 & 70 \\
\hline \multicolumn{3}{|l|}{ Cabello } \\
\hline En punta & 4 & 6 \\
\hline Muy Sombreado & 30 & 34 \\
\hline Normal & 82 & 55 \\
\hline Con raya al medio & 0 & 5 \\
\hline \multicolumn{3}{|l|}{ Omisión de manos y pies } \\
\hline $\mathrm{Si}$ & 14 & 40 \\
\hline No & 85 & 59 \\
\hline \multicolumn{3}{|l|}{ Paraguas } \\
\hline Presencia & 11 & 5 \\
\hline Ausencia & 88 & 95 \\
\hline \multicolumn{3}{|l|}{ Paraguas muy grande } \\
\hline de la persona dibujada & 3 & 2 \\
\hline Paraguas cubriendo & & \\
\hline adecuadamente a la persona & 5 & 2 \\
\hline $\begin{array}{l}\text { Paraguas muy chico respecto } \\
\text { al tamaño de la persona dibujad }\end{array}$ & la 3 & 6 \\
\hline \multicolumn{3}{|l|}{ Hombros } \\
\hline $\mathrm{Si}$ & 0 & 33 \\
\hline No & 100 & 67 \\
\hline Pies en direcciones puestas & 0 & 18 \\
\hline Pies descalzos & 0 & 31 \\
\hline Pies normales & 100 & 51 \\
\hline
\end{tabular}

Nota: la elaboración de la tabla es propia. Adaptado de American Psychological Association. Manual de publicaciones de la American Psychological Association ( $6^{\mathrm{a}}$ ed.). Mexico D.F.: Manual moderno 
Con respecto a las nubes en el dibujo los NNA realizaron dibujos con una notable presencia de nubes, de lo que se interpreta que estos se encuentran ante el temor de presión y amenaza producida por agentes externos, sin embargo en cuanto a los conflictos o aspectos conductuales hay ausencia de significado para este indicador.

La lluvia es otro indicador dentro de los resultados, pese a que en la consigna del test sea pedida la lluvia por el investigador o quien administre la prueba, por lo cual esto tiene un significado que viene asociado con el tipo de lluvia que los NNA realizaron, por tanto la lluvia indica que los NNA tienen temor de enfrentarse a un ambiente hostil, lo que muestra que los NNA sienten que no poseen los recursos suficientes, además la lluvia como agente estresor los imposibilita para enfrentar el ambiente porque el temor en ellos es mayor, lo anterior teniendo en cuenta que lo mayor parte de los NNA no dibujaron paraguas que indica que estos no poseen defensas suficientes. Por su parte los NNA que no dibujaron lluvia pese a la consigna del test, significa que estos NNA proyectan que tienen deseos de oponerse a lo que ocurre en su ambiente y a lo hostil que puede llegar a ser este y tienden a negar tanto a las presiones y conflictos de su entorno.

Ahora bien los tipos de lluvia que se presentaron en los dibujos fueron de tres tipos: escasa, torrencial y normal; de acuerdo con esto los NNA que realizaron lluvia escasa en su modalidad adaptativa indica que ellos poseen posibilidades de enfrentase a las presiones ambientales, de lo cual se infiere que estos niños tiene mayor disposición para adaptase al ambiente. En ese mismo sentido la lluvia normal no tiene significado alguno en el aspecto emocional; y la lluvia torrencial que es el otro tipo de lluvia hecho por los NNA muestra que existe mucha presión por tener que enfrentar un ambiente hostil y además de estrés y agobio por ese mismo entorno.

Las gotas como lágrimas en el dibujo de los NNA manifiesta que el conflicto representado por este indicador es la angustia. De la misma forma el indicador de botones, animales, flores y bolsillos indicador revela que estos NNA sienten temor por lo que acontece con su medio, incluyendo ambiente - población, temor que se manifiesta a través de la preocupación por lo social y preocupación somática, al igual se manifiesta dependencia y sentimientos de soledad por parte de los NNA, así mismo esta dependencia se manifiesta conductualmente en una búsqueda de protección, además de inmadurez emocional en los NNA, hecho que al estar estos en una etapa de cambios, es estereotipado que acontezca.

Así mismo el otro indicador que se presentó en los dibujos de los NNA es la omisión de manos, brazos y pies reflejando en temores que los NNA durante un ambiente hostil, presentan sensación de encierro e incomodidad, así mismo en los conflictos desvalorización, sentimiento de inadecuación, sentimiento de inferioridad, así como inadecuada percepción de sí mismo y dificultad para establecer relaciones con el mundo, en lo que se refiere a su aspecto conductual los temores y conflictos se muestran a través de la timidez, dependencia, retraimiento o agresividad.

Juntamente con lo anterior el porcentaje de NNA que omitió partes del cuerpo emplean como mecanismos de defensa la inhibición, lo que indica que estos NNA restringen, reprimen, aíslan o niegan ya sea una capacidad, función o conducta, con el fin de protegerse de otro tipo de temor que ocasionalmente es de tipo primario. Aunque el hecho de limitar una capacidad, función o conducta trae como consecuencia sufrimiento y disminución del funcionamiento de las estructuras Yoicas.

El paraguas es otro de los indicadores en el test persona bajo la lluvia en donde los dibujo de los NNA se caracterizaron por la ausencia de este en los dibujos, lo cual la ausencia de este predomino en la mayor parte de la población tanto en los NNA Mojana y San Jorge, esto indica que los NNA no poseen las defensas suficientes o herramientas para enfrentar un ambiente hostil como lo es la ola invernal u otro tipo de situación adversa, dicho esto desde el aspecto adaptativo que se activa ante la situación de presión ambiental.

Sin embargo solo un pequeño porcentaje de los NNA realizaron paraguas en sus dibujos, y estos paraguas fueron de tres tipos, el primero un paraguas muy grande con respecto al tamaño de la persona dibujada, el segundo tipo fue un paraguas cubriendo 
adecuadamente a la persona y por último un paraguas muy chico con respecto al tamaño de la persona dibujada. En ese orden el primer tipo de paraguas mencionado anteriormente indica que estos niños en cuanto a su modalidad defensiva poseen excesivas defensas y en su modalidad adaptativa expresan excesiva protección.

Así mismo el paraguas cubriendo adecuadamente a la persona solo un $2 \%$ y $5 \%$ de los NNA lo dibujaron, por tanto solo este porcentaje de NNA poseen defensas sanas, es decir que en su modalidad defensiva cuenta con los mecanismos de defensas y herramientas suficientes y adecuadas para enfrentar la presión que genera en ellos el ambiente, así mismo esto se ve reflejado adaptivamente porque es más fácil para ellos ajustarse a la realidad o entorno, puesto que confían en sí mismos, al igual que expresan sentimientos de adecuación y seguridad pese a las condiciones ambientales en las que se encuentran inmersos. El tercer tipo de paraguas que dibujaron los NNA fue el paraguas muy chico con respecto al tamaño de la persona dibujada, lo cual en relación a su modalidad defensiva, significa que presentan defensas débiles, es decir que no son lo suficientemente apropiadas para enfrentar su ambiente.

Ahora bien la información arrojada por los tests en este indicador muestra que los NNA hicieron dibujos donde la persona se observaba con hombros y con figura de mucha musculatura, lo cual exterioriza que estos NNA muestran una fachada de sobrecompensación, pero lo que realmente sienten son sentimientos de inseguridad e inadaptación, los cuales ocultan.

Los NNA hicieron en sus dibujos personas con pies en direcciones opuestas, pies descalzos y pies normales, este último carente de significado emocional, en esa secuencia mencionada los pies en direcciones opuesta significa que estos NNA presentan ambivalencia sobre la autonomía, es decir que estos infantes se sienten conflictuados a la hora de decidir entre ser autónomos o no serlos, presentado ellos entonces dificultades al adaptarse; así mismo el dibujar pies descalzos significa que estos NNA tienen deseos por mantenerse infantiles, puesto que al serlos se ven eximidos de responsabilidades, y ellos según la significación de este test no quieren realizar esfuerzos, por ello es el deseo de poder quedarse en esta etapa del desarrollo; hecho que afecta su acomodación a la realidad.

\section{DISCUSIONES}

Dado a las insuficientes investigaciones sobre el estado emocional en niños, niñas y adolescentes afectados por eventos climáticos, en este caso las inundaciones, se hace un tanto difícil poder contrastar los resultados obtenidos en este trabajo investigativo con otras investigaciones. Sin embargo la metodología utilizada permitió dar salida a los objetivos establecidos, las técnicas y procedimientos pueden ser utilizados para alcanzar estos resultados en otros contextos. Además, con esto se puede mostrar que las técnicas proyectivas son una herramienta útil en el diagnóstico del estado emocional en niños, niñas y adolescentes.

En este sentido los anteriores resultados pueden contrastarse con la investigación producto del diagnóstico realizado por Galindo et al. (2011) En el que se pudo evidenciar que los niños presentaban pasividad frente a la catástrofe (inundación del 2009, en el sur del atlántico), y prevalencia de mecanismos de defensa tales como la negación y el aislamiento, concluyendo así que estos niños al estar frente a una catástrofe que sobrepasa sus estrategias psicológicas responden de manera defensiva primitiva que entorpecen su sano desarrollo psicoafectivo. Así mismo, Díaz et al. (2010) encontraron que los síntomas emocionales que se identificaron en los momentos cercanos al paso del huracán fueron, la tristeza, el llanto, la labilidad afectiva, el desconsuelo y el miedo, que se relacionaron con preocupaciones por la integridad física de alguno de los padres que se encontraba ausente en esos momentos.

De igual manera, García (2011), al hacer el análisis de las reacciones, las cogniciones y las conductas que presenta la población adolescente entre los 12 y 16 años en la ciudad de Castellón ante la ocurrencia de un suceso desastroso o ante una emergencia, aunque se encuentre geográficamente del lugar del suceso, encontró que un mes después los sujetos continúan sintiendo tristeza, vulnerabilidad, indignación, miedo, rabia, culpabilidad, desgana e insumió, con mayor prevalencia en comparación con otras afectaciones que fueron disminuyendo. 
Ahora bien, los resultados de nuestra investigación muestran diferencias cuando se contrasta con la investigación de J.J. López y López (2011), siendo esta una investigación en la que si se patologiza el estado emocional de los niños, niñas y adolescentes porque el objetivo fue analizar la prevalencia del trastorno de estrés postraumático (TEPT) en menores de entre los 8 y 12 años. Cuya investigación mostró que el 55,4\% de los menores $(65,6 \%$ niñas y $46,9 \%$ niños) presentaba TEPT al mes del seísmo, y el 40,1\% (44,5\% niñas y $35,9 \%$ niños) al año. Una de cada dos niñas pequeñas (8-10 años) presenta TEPT al año del terremoto. No obstante, Guerra et al. (2014) desarrollaron una investigación cuyo objetivo fue evaluar la relación entre la magnitud percibida, el miedo experimentado, las creencias de autoeficacia y los síntomas de estrés post-traumático en adolescentes. Se esperaba que las creencias de autoeficacia se asociaran con los síntomas de estrés post-traumático y que esta relación fuera mediada por el miedo. Seis meses antes del terremoto, 218 adolescentes respondieron una escala de creencias de autoeficacia y tres meses después del terremoto reportaron la magnitud percibida del terremoto, el miedo experimentado y los síntomas de estrés posttraumático. La magnitud percibida no se asoció al miedo ni a los síntomas de estrés post-traumático, pero las creencias de autoeficacia y el miedo se asociaron con los síntomas de estrés post-traumático. Se encontró apoyo para el rol mediador del miedo percibido en la relación entre creencias de autoeficacia y síntomas de estrés post-traumático. Estos resultados sugieren que la prevención orientada a preparar a los adolescentes para enfrentar desastres naturales puede, además de salvar vidas, mejorar la respuesta emocional de aquellos que sobreviven.

En el marco de la investigación "Impacto del paso de los huracanes Gustav e Ike en la salud psicológica de un grupo de escolares afectados" (p. 508) desarrollada por Santiesteban et al. (2010), se evaluó el impacto de los huracanes Gustav e Ike en la salud psicológica de un grupo de escolares. Así mismo se caracterizó la representación psicológica que de los huracanes tuvieron estos, e identificó la presencia de síntomas psicológicos durante y posterior al paso de los meteoros por la localidad afectada. Los instrumentos utilizados fueron el dibujo libre, la composición con el tema "El ciclón", y la entrevista, además se realizó una entrevista a los padres, para recoger el comportamiento de los niños durante y después del paso del ciclón. Dentro de los resultados más relevantes se consideraron 3 categorías como representación psicológica, las cuales fueron destrucción, afección emocional y recuperación; y los síntomas identificados fueron la ansiedad y la tristeza, en 2 niños los síntomas fueron suficientes para considerar la posibilidad Trastorno de Adaptación con Síntoma Ansioso-depresivo.

En las investigaciones contrastadas se muestran algunas similitudes entre los resultados de una y otra, lo que indica que la investigación puede presentar un grado de confiabilidad en cuanto a resultados.

El presente estudio da cuenta del impacto en el estado emocional de niños, niñas y de adolescentes expuestos a las inundaciones durante el año 2011. Con respecto a esto, se puede decir que los resultados sugieren la pronta intervención a la población que ha sido afectada por desastre natural, específicamente con niños, niñas y adolescentes, con el objetivo de promover y mejorar el bienestar psicológico de estos. Así, de tal forma, se contribuye a que los conflictos $\mathrm{y}$ temores internos manifestados sean resueltos de manera significativa y consecuentemente que ayude en el desarrollo de conductas socialmente adaptativas. En el marco del trabajo de intervención para esta población afectada teniendo en cuenta los resultados se deberían desarrollar habilidades para la vida, habilidades resilientes e inteligencia emocional.

A partir de los resultados encontrados en cuantos a los temores, conflictos y la manifestación de tipo conductual de estos, así como la modalidad adaptiva y defensiva empleada por los niños, niñas y adolescentes afectados por el invierno, puede preguntarse y decirse si estos resultados son producto del invierno o de otras situaciones estresantes, adversas e importantes en el ciclo vital de los NNA afectados que puede distar de la emergencia de ola invernal y la situación de desastre que queda después de ella.

Teniendo en cuenta los referentes nacionales, y dada a la escasez de investigaciones cuyo objetivo de investigación estuviese enfocado a población infantil y adolescente en situación de desastres naturales, específicamente producto de inundaciones, se sugiere 
que la actividad investigativa se enfoque en estudiar a este tipo de población en estas situaciones para poder brindar el apoyo que se requiere a nivel psicosocial, teniendo en cuenta que Colombia es el tercer país con mayor presencia de desastres naturales y que solo dos investigaciones se habían hecho a nivel nacional entorno a esta problemática y población. Es por ello que la atención a la salud psicológica debe focalizarse en las consecuencias que provocan el estar expuestos a eventos climáticos en especial en la población infantil $\mathrm{y}$ adolescente.

De igual forma este estudio aporta conocimiento a la psicología de las emergencias, la cual ha sido poco abordada en nuestro contexto, en este sentido se tiene entonces conocimiento de las afectaciones emocionales y de los distintos cambios presentados en los NNA afectados por el invierno, además de como los NNA exteriorizan el conflicto que se generó en su psique producto del fenómeno invernal, al igual brinda herramientas para la intervención psicológica a los NNA afectados, tanto desde una perspectiva clínica y como también psicosocial, además de la realización de acciones de prevención de la emergencia, durante y después de esta, todas estas especialmente en individuos afectados por inundaciones.

Por otro lado se denota la valía de la utilización de instrumentos proyectivos para realizar investigaciones de este tipo, puesto que no se centran simplemente en el conflicto del individuo evaluado, sino además de las capacidades resilientes de estos, pero también aporta proyecciones de su personalidad, que son inconscientes para el mismo individuo, resaltando así el valor de estas para la elaboración de un psicodiagnostico. Por tanto la idea o mito de que instrumentos proyectivos y en este caso el test persona bajo la lluvia no son adecuados para realizar investigaciones con muestras poblaciones grandes queda sin fundamentos, puesto que queda demostrado que si es viable y que además el instrumento no determina la metodología de la investigación, si no la pretensión de los investigadores u objetivos de investigación; al igual queda claro que este instrumento si es útil y permite describir el estado emocional de NNA Afectados por el invierno, lo cual muestra que la técnica y procedimiento pueden ser utilizados en otros contextos, puesto que la metodología para este caso dio salida a los objetivos.
Igualmente como este instrumento permite resaltar las capacidades resilientes de los NNA afectados, se infiere que deberían utilizarse más instrumentos proyectivos para no tender a psicopatologizar la conducta de individuos afectados por algún tipo de desastres naturales, hecho que es una tendencia en las investigaciones que se han ejecutado en torno a esta problemática, por el tipo de instrumento que utilizan.

\section{REFERENCIAS}

Cardozo, A., Martínez, M. \& Colmenares, G. (2012). Caracterización de las condiciones de vida y el bienestar psicológico de adolescentes damnificados del sur del departamento del Atlántico. Revista científica Cultura, Educación, Sociedad - CES. 3(1), 83-106. Recuperado de http://revistascientificas.cuc.edu.co/index.php/ revistaces/article/view/161

Ministerio de la protección social. (2006). Ley 1098, Código de la infancia y adolescencia. Recuperado de http://www.icbf.gov.co/portal/page/portal/ PortalICBF/Bienestar/LeyInfanciaAdolescencia/ SobreLaLey/CODIGOINFANCIALey1098.pdf

Comisión Económica para América Latina y el Caribe (CEPAL). 2012. Valoración de daños y pérdidas. Recuperado de http://www.cepal.org/colombia/ noticias/documentosdetrabajo/7/49587/Libro_ Ola_invernal_en_Colombia_BID_CEPAL.pdf

Presidencia de la república (2011).Constitución política de Colombia de 1991. http://wsp.presidencia.gov. co/Normativa/Documents/ConstitucionPoliticaC olombia_20100810.pdf

Corte constitucional, (2014). Acción de tutela de personas afectadas por desastres naturales. República de Colombia. Recuperado de http:// www.corteconstitucional.gov.co/relatoria/2014/ T-198-14.htm

Guerra, C., Cumsille, P. \& Martínez M. L. (2014). Post-traumatic stress symptoms in adolescents exposed to an earthquake: Association with self-efficacy, perceived magnitude, and fear, International Journal of Clinical and Health Psychology, Volume 14(3), 202-207. Recuperado de http://dx.doi.org/10.1016/j.ijchp.2014.05.001.( 
http://www.sciencedirect.com/science/article/pii/ S1697260014000118)

Chóliz, M., (2005). Psicología de la emoción: el proceso emocional. Recuperado de http://www. uv.es/=choliz/Proceso $\% 20$ emocional.pdf

DANE. (2011). El DANE entregó resultado definitivo del Registro Único de Damnificados 2010-2011. Recuperado de http://www.dane.gov.co/files/ noticias/Resultados_Finales_Reunidos.pdf

Dobles, C., Zúñiga, M. \& García, J. (1998). Investigación en educación: procesos, interacciones y construcciones. Recuperado de http://www.researchgate.net/profile/ Luis_Meza6/publication/26494042_ Metodologa_de_la_investigacin_educativa_ Posibilidades_de_integracin_culturales/links/ 5519d0d20cf26cbb81a2b2f9.pdf

Díaz, C. A., Quintana, G. R. \& Vogel, E. H. (2012). Síntomas de depresión, ansiedad y estrés posttraumático en adolescentes siete meses después del terremoto del 27 de febrero de 2010 en Chile. Revista Terapia psicológica, 30(1), 3743. Recuperado de http://www.scielo.cl/scielo. php?script=sci_arttext\&pid=S0718

Díaz, Y.S., Castro, M. \& González, Z. (2010).Impacto del paso de los huracanes Gustav e Ike en la salud psicológica de un grupo de escolares afectados. Revista Cubana de Medicina General Integral, 26(3), 508-515. Recuperado de http://scielo.sld. cu/pdf/mgi/v26n3/mgi08310.pdf

Philosophica. (2007). Enciclopedia filosófica on line. Recuperado de URL:http://www.philosophica. info/archivo/2007/voces/emociones/Emociones. html

Freud, A., (1980). El Yo y los mecanismos de defensa. Barcelona: Paidos Ibérica.

Freud, S., (1915). The Psychopathology of Everyday Life. New York: Holt.

Galindo, J., Pacheco, K., \& Russo, A. (2011). Efectos psicosociales y psicoafectivos generados en las poblaciones afectadas por las inundaciones en el sur del Atlántico durante el año 2010. Revista Cultura Educación y Sociedad-CES, 2(1), 8999. Recuperado de http://www.revistascientificas. cuc.edu.co/index.php/revistaces/article/.../pdf_29
Galor, S. \& Hentschel, U. (2013). El uso de los mecanismos de defensa como herramientas de afrontamiento por veteranos israelíes deprimidos y con TEPT. Revista Subjetividad y Procesos Cognitivos, 17(1), 118-133. Recuperado de http:// www.redalyc.org/articulo.oa? $\mathrm{id}=339630261005$

García Renedo, M. (2011). Reacciones de adolescente antedesastresyemergencias:aspectospsicosociales derivados del 11 de marzo. (Tesis doctoral, universitat Jaume I). Recuperado de http://www. tdx.cat/bitstream/handle/10803/10526/grenedo. pdf; jsessionid=503CD518B1D91CB5729DA53 CC842398D.tdx 1 ? sequence $=1$

Guimaro, M. S., Caiuby, A. V. S., Dos Santos, O. F. P., Lacerda, S. S., \& Andreoli, S. B. (2013). Sintomas de estresse pós-traumático em profissionais durante ajuda humanitária no Haiti, após o terremoto de 2010. Revista Ciência \& Saúde Coletiva, 18(11), 3175-3181. Recuperado de http://www.redalyc. org/articulo.oa?id=63028795008

Informe ola Invernal (2011). Departamento de sucre, ola invernal 2010. Recuperado de http://www. slideshare.net/legilelu/informe-ola-invernalsucre-2011

Leiva Bianchi, M., Baher, G. \& Poblete, C. (2012). The Effects of Stress Coping Strategies in PostTraumatic Stress Symptoms Among Earthquake Survivors: An Explanatory Model of PostTraumatic Stress. Revista Terapia psicológica, 30(2), 51-59. Recuperado de http://www.scielo. cl/scielo.php?script=sci_arttext\&pid=S0718-

Loubat, M., Fernández, A. M. \& Morales, M. (2010). La Experiencia de Peralillo: Una Intervención Psicológica para el Estado de Emergencia. Revista Terapia Psicológica, 28(2), 203-207. Recuperado de http://www.redalyc.org/articulo. oa? $\mathrm{id}=78516731009$

López-García,J.\& López-Soler,C.(2013).Trastorno de estrés postraumático en escolares tras el terremoto de Lorca (España) en 2011. Revista Elsevier España, S.L., 28(3), 230-233. Recuperado de http://scielo.isciii.es/scielo.php?script=sci_arttext \&pid=S021391112014000300010\&lng=es.http:// dx.doi.org/10.1016/j.gaceta.2013.10.009 
Maldonado, M.L. (2002). Test persona bajo la lluvia. MLM Consultoras. Recuperado de http:/www. infogerontologia.com/documents/miscelanea/ test_evaluacion/testpersonabajolalluvia.pdf

DANE, (2005). Necesidades Básicas Insatisfechas por total, cabecera y resto, según departamento y el país, resultados Censo General. Recuperado de http://www.dane.gov.co/censo/files/resultados/ NBI_total_30_Jun_2011.pdf

Observatorio del Caribe Colombiano - PNUD (2008). El Caribe Colombiano frente a los objetivos del desarrollo del milenio. Recuperado de http:// www.pnud.org.co/img_upload/33323133323161 646164616461646164/LINEA\%20DE\%20BASE \%20ODM\%20CARIBE.pdf
Gobernación de sucre (2008). Plan de desarrollo departamental de sucre (2008-2011). Recuperado de http://www.sucre.gov.co/apcaafiles/303 $33365656132656531383331643435 / \mathrm{Plan}$ Departamental_de_Desarrollo_2008__2011.pdf

Ocaribe (2012). Plan de desarrollo departamental de sucre (2012-2015). Recuperado de, http://www. ocaribe.org/cargar_imagen.php?tipo $=22 \& i d=49$

Portuondo (1971). La figura humana test proyectivo de Karen Machover. Madrid: Biblioteca Nueva. Recuperado de http://thevalle323.xtrweb.com/ test-psicologia-vallevallester/Test\%20Proyectivo \%20de\%20Karen\%20Machover\%20By\%20Luis $\% 20$ Vallester\%20.pdf

Fecha de recepción: 29 de marzo de 2016

Fecha de aceptación: 29 de abril de 2016 